\title{
Reversible barocaloric effects over a large temperature span in fullerite $\mathbf{C}_{60}$ Supplementary Material
}

\author{
Junning $\mathrm{Li}^{1}$, David Dunstan ${ }^{1}$, Xiaojie Lou ${ }^{2}$, Antoni Planes ${ }^{3}$, Lluís \\ Mañosa $^{3}$, María Barrio ${ }^{4}$, Josep-Lluís Tamarit ${ }^{4}$, and Pol Lloveras ${ }^{4}$ \\ ${ }^{1}$ School of Physics and Astronomy, Queen Mary University of London, London E1 4NS, United \\ Kingdom \\ ${ }^{2}$ Frontier Institute of Science and Technology and State Key Laboratory for Mechanical \\ Behavior of Materials, Xi'an Jiaotong University, Xi'an 710049, China \\ ${ }^{3}$ Departament de Fúsica de la Matèria Condensada, Facultat de Física, Universitat de \\ Barcelona, Martí i Franquès 1, 08028 Barcelona, Catalonia \\ ${ }^{4}$ Grup de Caracterizació de Materials, Departament de Fúsica, EEBE and Barcelona Research \\ Center in Multiscale Science and Engineering, Universitat Politècnica de Catalunya, Eduard \\ Maristany, 10-14, 08019 Barcelona, Catalonia
}




\section{X-ray diffraction}
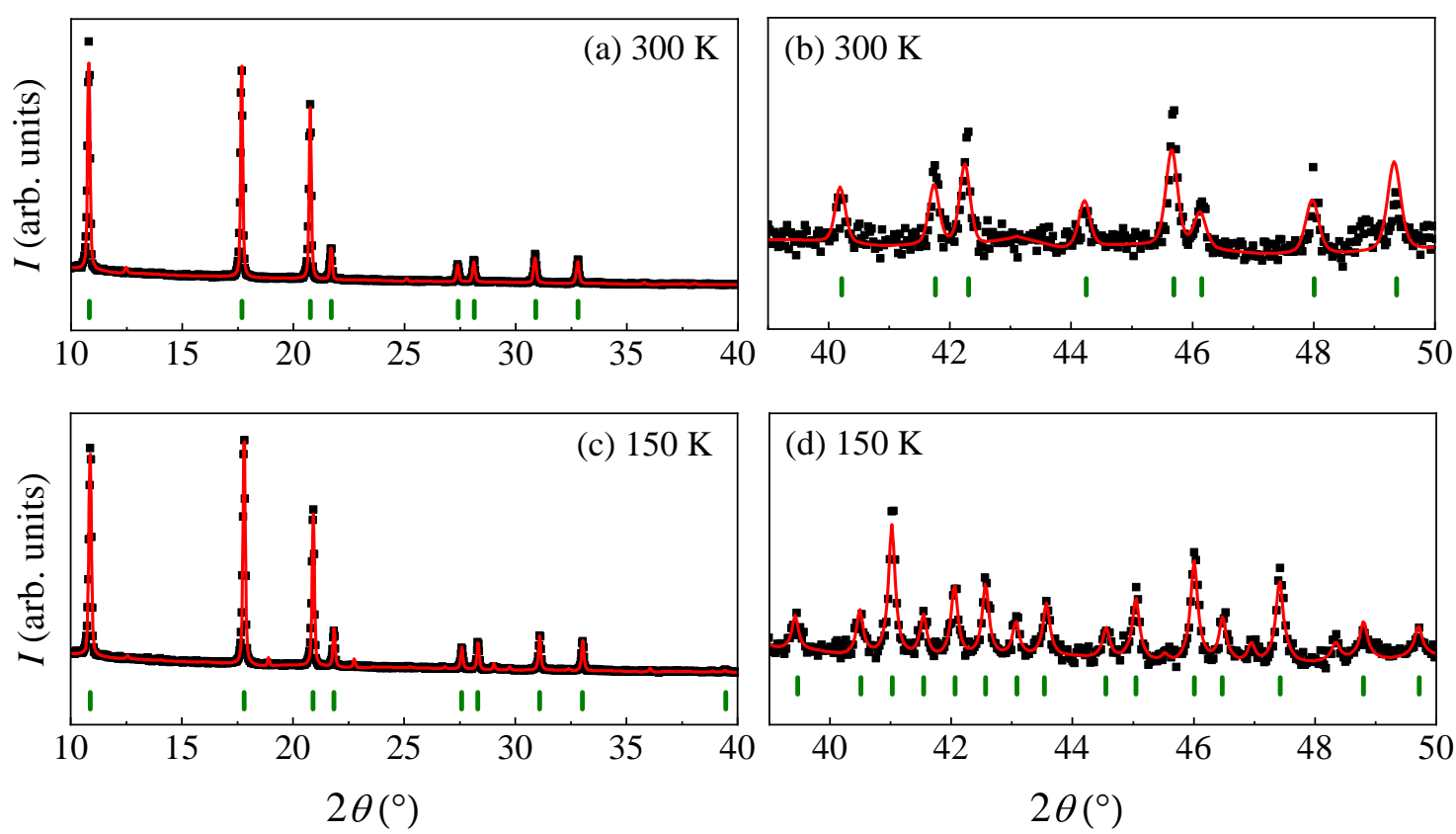

Figure S1: Diffraction patterns in $(a, b)$ the fcc phase and $(c, d)$ the simple cubic phase, in the angle ranges $(\mathrm{a}, \mathrm{c}) 2 \theta \in(10,40)^{\circ}$ and $(\mathrm{b}, \mathrm{d}) 2 \theta \in(39,50)^{\circ}$ (different vertical scales in each panel). Black symbols correspond to experimental data, red lines are calculated patterns via pattern matching fitting procedure and green lines indicate the position of the Bragg peaks. 
Table S1: Angular positions and the corresponding Miller indices (hkl) for $\mathrm{C}_{60}$ for the two phases at the indicated temperatures.

\begin{tabular}{|cc|cc|}
\hline \multicolumn{2}{|c|}{ fcc $(T=300 \mathrm{~K})$} & \multicolumn{2}{|c|}{ simple cubic $(T=150 \mathrm{~K})$} \\
\hline $2 \theta\left(^{\circ}\right)$ & $\mathrm{hkl}$ & $2 \theta\left(^{\circ}\right)$ & hkl \\
\hline 10.8196 & $(111)$ & 10.8812 & $(111)$ \\
17.6871 & $(220)$ & 17.7987 & $(220)$ \\
20.7672 & $(311)$ & 20.8996 & $(311)$ \\
21.7046 & $(222)$ & 21.8415 & $(222)$ \\
27.4145 & $(331)$ & 27.5954 & $(331)$ \\
28.1404 & $(420)$ & 28.3207 & $(420)$ \\
30.8994 & $(422)$ & 31.1028 & $(422)$ \\
32.8131 & $(511,333)$ & 33.0294 & $(511)$ \\
40.2168 & $(620)$ & 39.4687 & $(532)$ \\
41.7640 & $(533)$ & 40.5076 & $(620)$ \\
42.3060 & $(622)$ & 41.0248 & $(621)$ \\
44.2484 & $(444)$ & 41.5466 & $(541)$ \\
45.6942 & $(711,551)$ & 42.0661 & $(533)$ \\
46.1570 & $(640)$ & 42.5630 & $(622)$ \\
48.0090 & $(642)$ & 43.0824 & $(542)$ \\
49.3642 & $(553)$ & 43.5342 & $(631)$ \\
& & 44.5505 & $(444)$ \\
& & 45.0474 & $(362)$ \\
& & 46.0091 & $(551)$ \\
& & 46.4704 & $(640)$ \\
& & 47.4230 & $(633)$ \\
& & 48.7967 & $(544)$ \\
& & 49.7228 & $(731)$ \\
& & 50.6036 & $(463)$ \\
& & 51.0779 & $(650)$ \\
& & & $(470)$ \\
\hline
\end{tabular}




\section{Variable-pressure calorimetry at different temperature rates}

Measurements of heat flow $d Q /|d T|$ at atmospheric pressure performed using the commercial Q100 (TA Instruments) at different temperature rates from 1 to $10 \mathrm{~K} \mathrm{~min}^{-1}$ (see Fig. S2) show that both endothermic and exothermic onset transition temperatures are basically rate-independent and therefore hysteresis is also rate-independent. Endothermic and exothermic transition temperature peaks are slightly shifted to higher and lower temperatures, respectively, due to the fact that the temperature is measured at the inert reference.

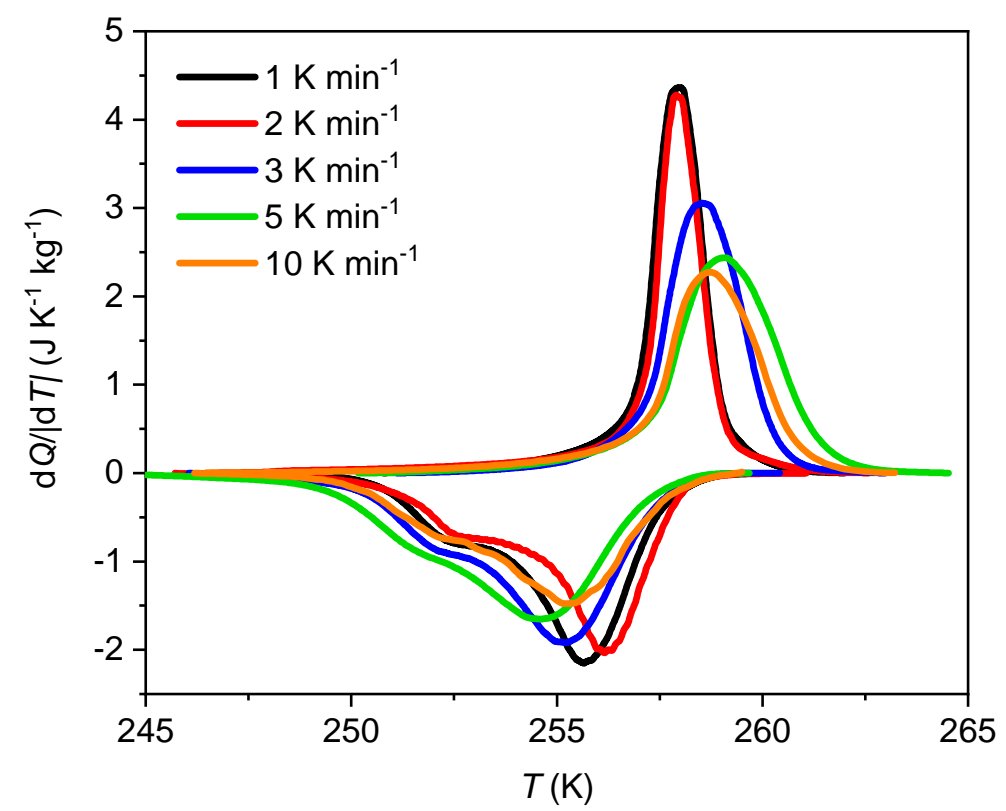

Figure S2: Heat flow in temperature measured by commercial Q100 (TA Instruments) across the endothermic (positive) and exothermic (negative) transitions at different temperature rates, after baseline subtraction.

Figure S3 shows a comparison of heat flow measurements performed across the endothermic transition using the three different calorimeters at different temperature rates. It can be seen that in calorimeters Q100 and A, the onset transition temperature is basically independent of $\dot{T}$ whereas in calorimeter $\mathrm{B}$ it does depend on $\dot{T}$. This artifact arises because the thermocouple measuring temperature in calorimeter $\mathrm{B}$ is placed at a certain distance from the sample and closer to the thermal jacket of the high-pressure cell. This introduces a delay between the measured temperature and sample temperature such that, given the same remaining experimental conditions, the higher $\dot{T}$, the larger the difference between the two quantities. Our variable-pressure measurements on heating used for the calculations of the barocaloric effect have been performed at the highest rate allowed by the thermal bath $\left(\dot{T} \sim 5 \mathrm{~K} \mathrm{~min}^{-1}\right)$. While this rate introduces a larger uncertainty in temperature, it gives rise to a smoother baseline, which leads to a 
lower uncertainty in the determination of the transition entropy changes. With respect to cooling ramps, maximum absolute temperature rates allowed by the thermal bath are much lower $\left(|\dot{T}| \sim 2 \mathrm{~K} \mathrm{~min}^{-1}\right)$ than heating ramps, which introduces much smaller errors in the exothermic transition temperatures measured by calorimeter B than those corresponding to the endothermic ones. Variations in the barocaloric response originating in this fact are within the error reported in the main text and therefore do not affect the results nor conclusions of the present study.

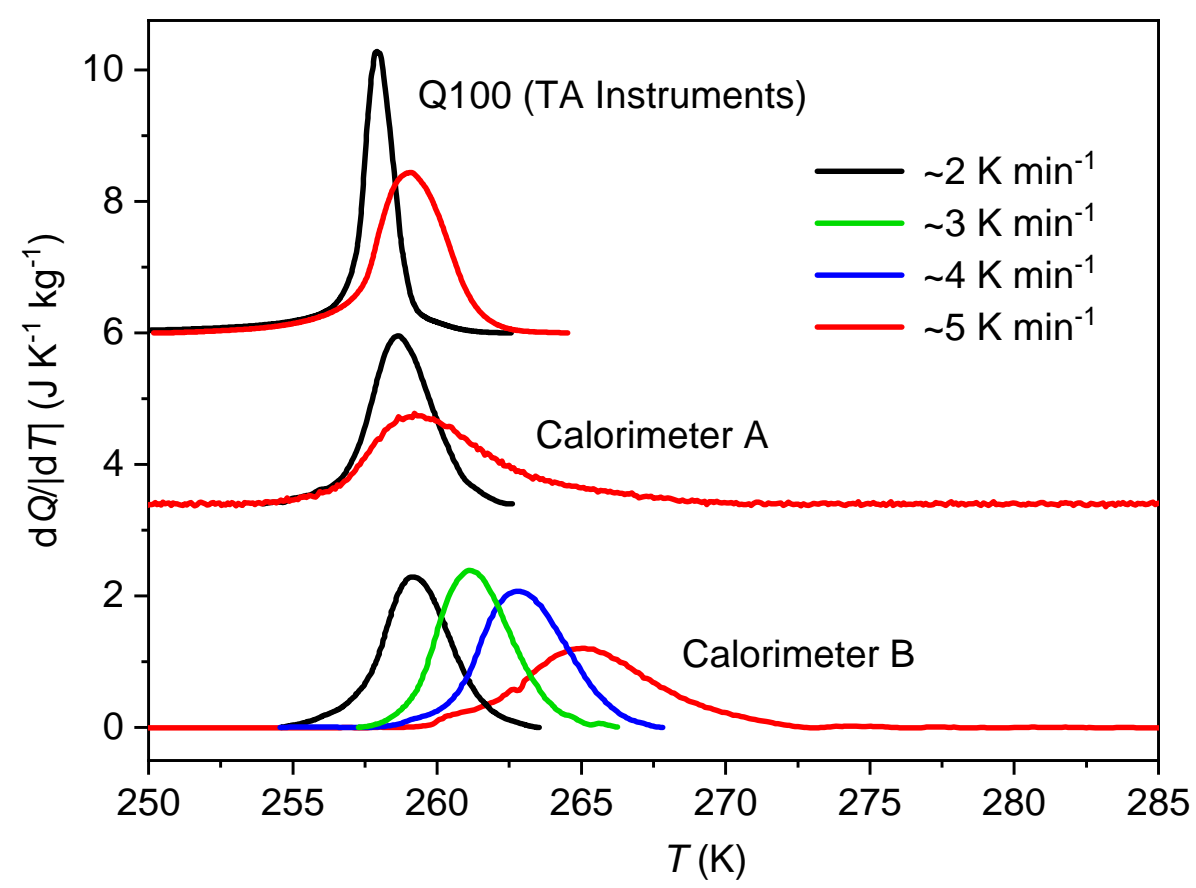

Figure S3: Heat flow in temperature measured by the three different calorimeters at atmospheric pressure and at different temperature rates, after baseline subtraction. Peaks have been shifted vertically for clarity. 


\section{Construction of the heat capacity at different pressures in each phase}

In this section, we provide a more detailed explanation for the construction of the temperature- and pressure-dependent heat capacity. First, in Fig. S4 we reproduce temperature- and pressure-dependent volume data for the fcc phase provided by Ref. [1] (black lines and symbols). On top of these data we have plotted red straight lines with the slope $\left(\frac{\partial V}{\partial T}\right)_{p_{\text {atm }}}$ as determined from our x-ray measurements at atmospheric pressure in the temperature range (265-360) K (see Fig. 1 in the main article). The good agreement between the two sets of data in the temperature range of interest allows to reasonably assume that

$$
\left(\frac{\partial V}{\partial T}\right)_{p} \sim\left(\frac{\partial V}{\partial T}\right)_{p_{\text {atm }}}
$$

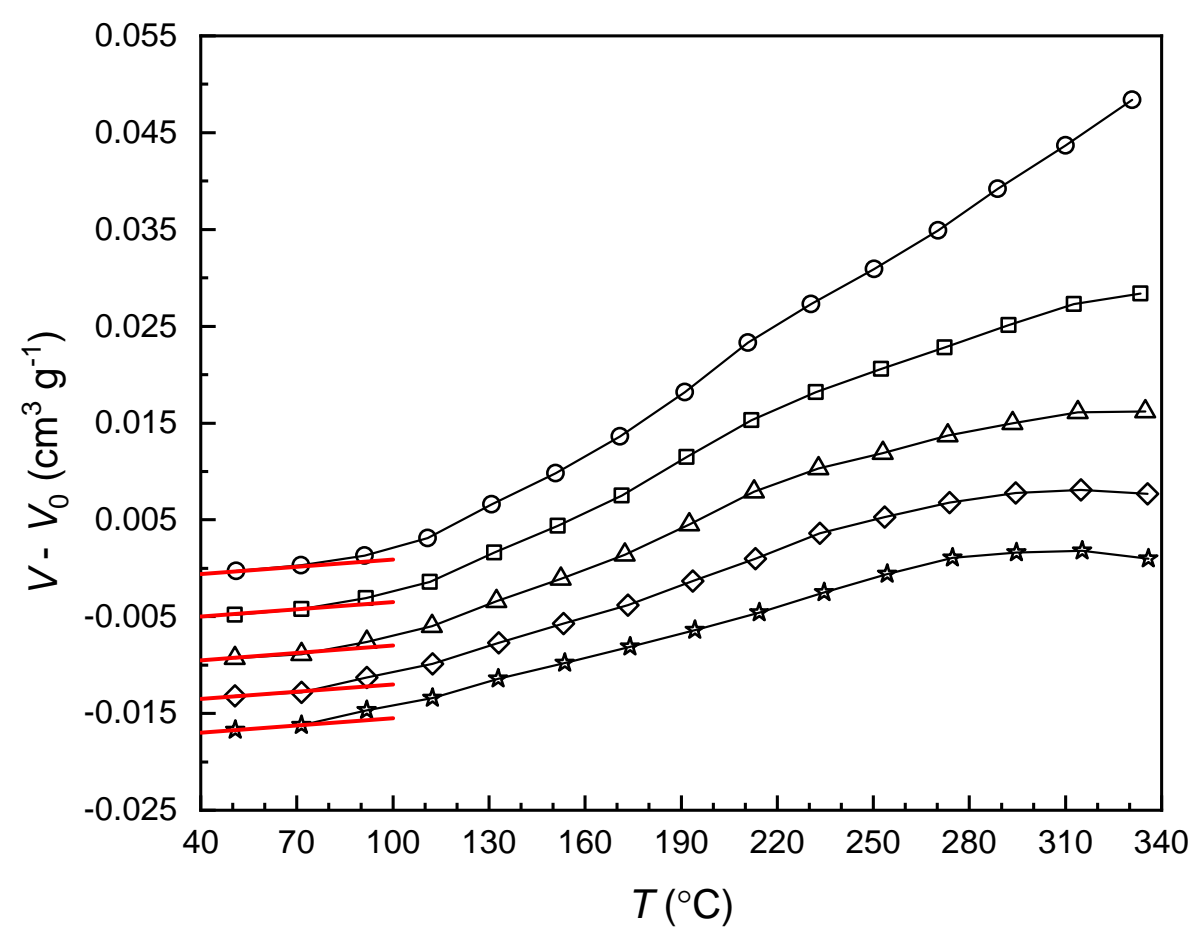

Figure S4: Black symbols and lines: Temperature- and pressure-dependent specific volume from literature data [1]. From top to bottom, literature data is measured at 10, 50, 100, 150 and $200 \mathrm{MPa}$. Red straight lines have the slope $\left(\frac{\partial V_{\mathrm{fcc}}}{\partial T}\right)_{p_{\text {atm }}}$ as obtained by our x-ray diffraction measurements at atmospheric pressure.

According to Fig. If of the main article, $V(T)$ obtained from our x-ray measurements reveals different behavior in four temperature intervals: For $T \in(150,250) \mathrm{K}$ and $T \in$ $(265,360) \mathrm{K}, V(T)$ is linear; for $T \in(250,260) \mathrm{K}, V(T)$ is nonlinear; and around the 
transition $V(T)$ is nearly discontinuous. Then, given the assumption in Eq. S1, we can construct $V(T)$ plot at a higher pressure $p$ such that all the mentioned regimes are shifted to higher temperatures an amount given by $\frac{d T}{d p}\left(p-p_{\text {atm }}\right.$ ) (as represented by the black arrow between the peaks in heat flow at two different pressures displayed in Fig. $\mathrm{S} 5 \mathrm{a}$ ). Here $\frac{d T}{d p}$ is the transition temperature shift determined experimentally (see Fig. $2 \mathrm{~b}$ in the main article). Resulting qualitative $V(T)$ curves at two different pressures are shown in Fig. S5b, where dashed lines separate linear and nonlinear $V(T)$ regions.

From these curves, the following Eq. S2:

$$
\left(\frac{\partial C_{p}}{\partial p}\right)_{T}=-T\left(\frac{\partial^{2} V}{\partial T^{2}}\right)_{p}
$$

can be used to establish the dependence of the heat capacity on pressure, from literature data [2]. Therefore, in the temperature intervals where $V(T)$ is linear, $C_{p}$ is independent of pressure whereas in the temperature intervals where $V(T)$ is not linear, $C_{p}$ depends on pressure (see Fig. S5c). In the latter region, $V(T)$ appears to be concave, which means that $\left(\frac{\partial^{2} V}{\partial T^{2}}\right)_{p}>0$. This means that, according to eq. S2, $C_{p}$ decreases when increasing pressure and the decrease is larger at higher temperature. This feature can be seen qualitatively in the region $T_{1}<T<T_{2}$ in Fig. S5c. 


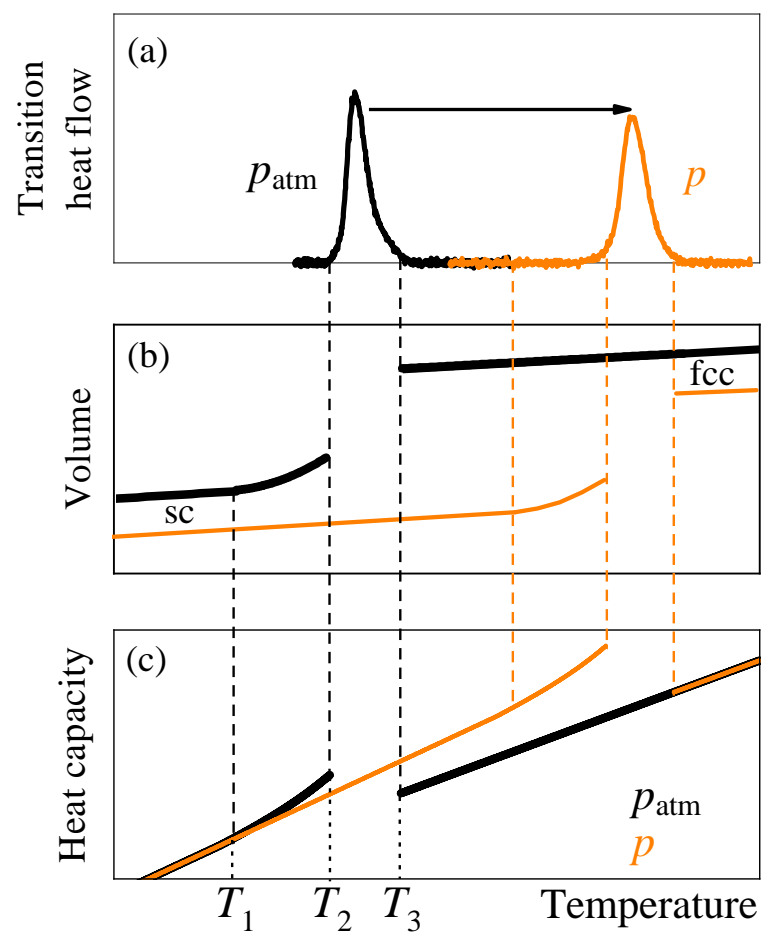

Figure S5: Qualitative plots for the construction of the heat capacity. (a) Temperaturedependent transition heat flow measured at $p_{\text {atm }}$ and $p$, after baseline subtraction. (b) Temperature-dependent volume at two different pressures, $p_{\text {atm }}$ and $p$, assuming $\left(\frac{\partial V_{\text {fcc }}}{\partial T}\right)_{p} \sim\left(\frac{\partial V_{\text {fcc }}}{\partial T}\right)_{p_{\text {atm }}}$. (c) Temperature-dependent heat capacity at $p_{\text {atm }}$ and $p$, constructed from data in panels $(\mathrm{a}, \mathrm{b})$. 


\section{References}

[1] W. Li, R. D. Sherwood, D. M. Cox, and M. Radosz, J. Chem. Eng. Data, 1994, 39, 467-469.

[2] V. V. Diky, L. S. Zhura, A. G. Kabo, V. Y. Markov and G. J. Kabo, Fullerene Sci. Techn., 2001, 9, 543-551. 